\title{
Propagation of nuclear data uncertainties for fusion power measurements
}

\author{
Henrik Sjöstrand ${ }^{1, a}$, Sean Conroy ${ }^{1}$, Petter Helgesson ${ }^{1}$, Solis Augusto Hernandez ${ }^{1}$, Arjan Koning ${ }^{1,2}$, Stephan Pomp ${ }^{1}$, \\ and Dimitri Rochman ${ }^{3}$ \\ ${ }^{1}$ Division of Applied Nuclear Physics, Department of Physics and Astronomy, Uppsala University, Box 516, 751 20, Uppsala, Sweden \\ 2 Nuclear Data Section, IAEA, Vienna, Austria \\ 3 Reactor Physics and Systems Behaviour Laboratory, Paul Scherrer Institut, Villigen, Switzerland
}

\begin{abstract}
Neutron measurements using neutron activation systems are an essential part of the diagnostic system at large fusion machines such as JET and ITER. Nuclear data is used to infer the neutron yield. Consequently, high-quality nuclear data is essential for the proper determination of the neutron yield and fusion power. However, uncertainties due to nuclear data are not fully taken into account in uncertainty analysis for neutron yield calibrations using activation foils. This paper investigates the neutron yield uncertainty due to nuclear data using the so-called Total Monte Carlo Method. The work is performed using a detailed MCNP model of the JET fusion machine; the uncertainties due to the cross-sections and angular distributions in JET structural materials, as well as the activation cross-sections in the activation foils, are analysed. It is found that a significant contribution to the neutron yield uncertainty can come from uncertainties in the nuclear data.
\end{abstract}

\section{Fusion yield measurements}

Fusion plasmas produce neutrons, and by measuring the neutron emission, the fusion power can be inferred. Accurate neutron yield measurements are paramount for the safe and efficient operation of fusion experiments, in particular with respect to the tritium accountancy [1]. At JET, a system of activation foils provides the absolute calibration for the neutron yield determination and a similar system is proposed for ITER. The activation system uses the property of certain nuclei to emit radiation after being excited by neutron reactions. A sample of suitable nuclei is placed in the neutron flux close to the plasma, and after irradiation the induced radiation is measured. Knowing the neutron activation cross-section one can calculate the time-integrated neutron flux at the sample position. To relate the local flux to the total neutron yield, the spatial flux response has to be identified. This describes how the local neutron emission affects the flux at the detector. The required spatial flux response is commonly determined using neutron transport codes, e.g., MCNP.

Nuclear data is used as input both in the calculation of the spatial flux response and when the flux at the irradiation site is inferred. Consequently, high-quality nuclear data is essential for the proper determination of the neutron yield and fusion power. In particular, the uncertainties due to nuclear data stems from two sources:

1) The uncertainty in the nuclear data of the activation system. i.e., the propagated uncertainties due to nuclear data in the activation reaction to the local neutron flux. In this work, the commonly used ${ }^{115} \operatorname{In}\left(n, n^{\prime}\right)^{115 m}$ In reaction is investigated.

a e-mail: henrik.sjostrand@physics.uu.se
2) The uncertainties in the neutron flux due to uncertainties in the nuclear data in the structural material of the fusion machine, in this case, JET. This nuclear data is normally referred to as transport nuclear data.

In this paper, the neutron yield uncertainty due to nuclear data is investigated using the so-called Total Monte Carlo Method, TMC.

\section{Method}

The reaction rate, $R$, per activation foil atom, is determined by the energy-dependent neutron flux, $\Phi(E)$, and the energy-dependent reaction cross-section, $\sigma(E)$, by Eq. (1).

$$
R=\int \sigma(E) \Phi(E) \partial E
$$

In order to get the reaction rate in the activation foil, $R$ has to be multiplied by the number of atoms in the activation foil. As can be seen in the equation the reaction rate is dependent both on the neutron spectrum and the absolute magnitude of the neutron flux. In order to study the effects of the cross-section, one collapses the cross-section to a so-called 'one group cross-section' also denoted effective cross-section in, e.g., Ref. [2]. The effective cross-section is the cross-section folded with the normalized neutron spectrum as illustrated in Eq. (2).

$$
\begin{aligned}
& \langle\sigma\rangle=\int \sigma(E) \Phi_{N}(E) \partial E \\
& \Phi_{N}(E)=\frac{\Phi(E)}{\int \Phi(E) \partial E}
\end{aligned}
$$

In terms of nuclear data, the reaction rate is dependent on two quantities. One is the activation cross-section, 
$\sigma_{a}=\sigma(E)$, which directly affects the reaction rate. The other is the transport nuclear data, $\sigma_{\Phi}$, i.e., the cross-sections and angular distributions of the nuclides in the structural materials in the JET machine; these affects the flux and consequently also the reaction rate. Since $R=f\left(\sigma_{a}, \sigma_{\Phi}\right)$ and $\sigma_{a}$ and $\sigma_{\Phi}$ is independent and for small uncertainties we can assume linearity; hence standard uncertainty propagation can be performed. i.e., the uncertainty contributions can be summed in square.

The uncertainty in $R$ due to transport data, $\partial R_{\sigma_{\Phi}}$, is actually also depending on the choice of activation reaction since the shape of the energy dependence of the activation reaction cross-section probes different parts of the neutron spectrum and hence probe different effects in the transport process. In this work $\partial R_{\sigma_{\Phi}}$ is calculated for the ${ }^{115} \operatorname{In}\left(n, n^{\prime}\right)^{115 m} \mathrm{In}$ and ${ }^{93} \mathrm{Nb}(n, 2 n)^{92 m} \mathrm{Nb}$ for the activation reactions, since these are the two important activation reactions for DD and DT operation respectively.

The uncertainty due to the activation cross-section, $\partial R_{\sigma_{a}}$, is directly proportional to the uncertainty in the effective cross-section. In this work, only the uncertainty in the ${ }^{115} \operatorname{In}\left(n, n^{\prime}\right)^{115 m}$ In reaction is investigated for activation uncertainties.

\subsection{The Total Monte Carlo method (TMC)}

In the TMC method, the TALYS based nuclear physics code system and experimental data are combined [3]. This combination of nuclear theory and experiment allows associating uncertainties to nuclear data (ND). Many different types of nuclear data can be analysed, such as transport data, e.g., cross-sections and angular distributions, and activation data.

TMC starts from the probability distributions of nuclear model parameters. These distributions are sampled, and a large set of random ND libraries (from now on referred to as random files) are created using TALYS. These ND files are subsequently processed into ENDF format. After some final processing, the accepted random files are used to obtain distributions in macroscopic quantities of the physical system under study. The final spread in the observed macroscopic parameter is interpreted as the systematic uncertainty due to ND. In many cases, however, there can also be a contribution due to statistics, e.g., in the case of using MCNP. Consequently, the variance due to statistics needs to be subtracted from the observed variance in order to obtain the variance in nuclear data [4].

The set of random files constitutes TENDL [5], the Talys Evaluated Nuclear Data Library, where the file that best represents experimental data is the central value of the TENDL library, and the spread of the random files are used to create the TENDL covariance matrix. The TENDL is updated yearly, and the different versions are referred to with their year of production, e.g., TENDL2015. Between TENDL2013 and TENDL2015 there has been a transition in methodology for calibrating the spread of the nuclear data, i.e., the ND covariance. Whereas in TENDL2013, more qualitative comparisons against experimental data were used; TENDL2015 uses more quantitative methods including Bayesian updating [6]. In this work, TENDL2015 data is used for the transport nuclear data, whereas TENDL2013 is used for the activation data.

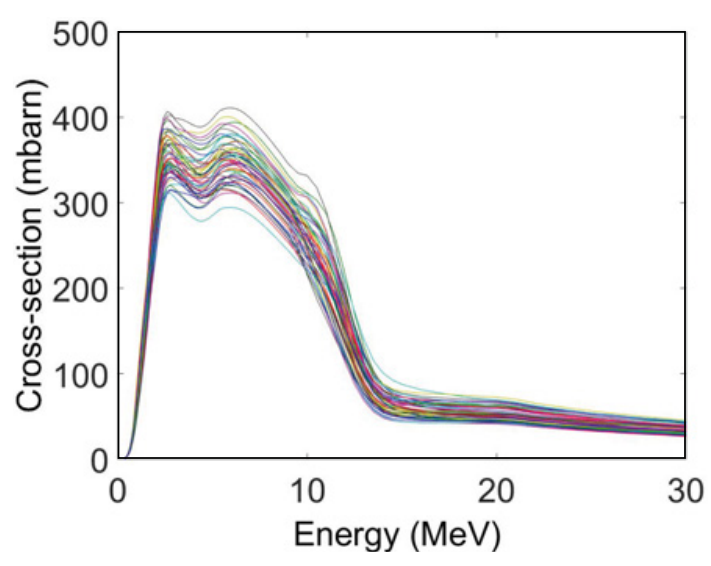

Figure 1. Plot of the distribution of random files describing the uncertainty of the ${ }^{115} \operatorname{In}\left(n, n^{\prime}\right)^{115 m} \operatorname{In}$ reaction.

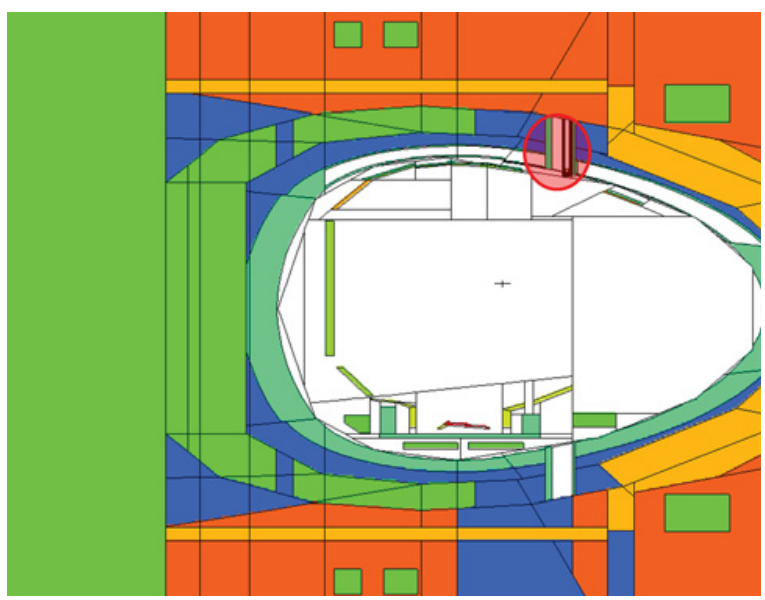

Figure 2. JET MCNP Model. The area encircled in red marks the location of the activation foils and was the focus of this investigation for the transport nuclear data.

\subsubsection{TMC for ${ }^{115} \operatorname{In}\left(n, n^{\prime}\right)^{115 m} / n$ effective cross-section}

For ${ }^{115} \operatorname{In}\left(n, n^{\prime}\right)^{115 m}$ In the central file (the best estimate) of TENDL2013 were normalized to IRDFF1.0. In total 50 random files, which spread around the central file and includes the co-variance information for ${ }^{115} \mathrm{In}$, have been produced. The results for the ${ }^{115} \operatorname{In}\left(n, n^{\prime}\right){ }^{115 m} \operatorname{In}$ are presented as energy- cross-section tables. The spread in the cross-section in the different random files are illustrated in Fig. 1.

\subsubsection{Propagating transport nuclear data uncertainties}

A JET MCNP model [2] was used to calculate the flux and the activation in the JET activation systems. Uncertainties for gamma fluxes, neutron fluxes, as well as activation coefficients (reaction rates) were calculated. The fluxes around the JET vertical port were primarily investigated. The MCNP model used is shown in Fig. 2.

In this work the following isotopes were varied: ${ }^{52} \mathrm{Cr}$, ${ }^{54,56} \mathrm{Fe},{ }^{58,60} \mathrm{Ni}$, and ${ }^{63,65} \mathrm{Cu}$, since these were the isotopes considered to have the largest impact in the nuclear data uncertainty. 300 random files were used for each isotope. As mentioned above, TENDL contains the uncertainty both in respect to the cross-sections in all relevant reaction 


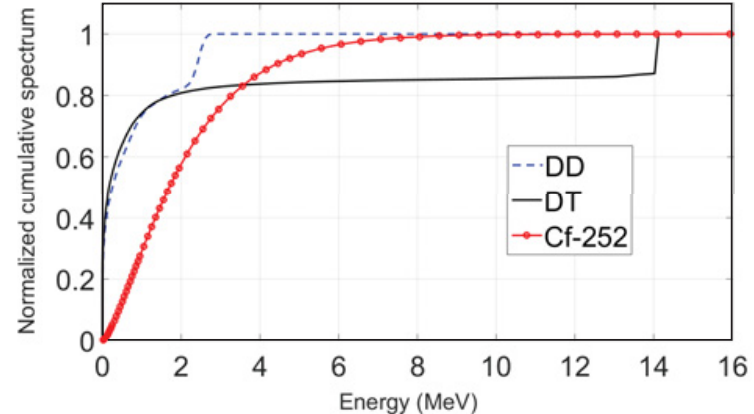

Figure 3. Cumulative normalized neutron spectra for DD, DT, and ${ }^{252} \mathrm{CF}$.

channels, including cross- correlations, as well as the angular distributions. Consequently, all these uncertainties, with correlations, are propagated for the above-listed isotopes.

The random ENDFs (random files) were obtained from the TENDL webpage [5] and processed with NJOY to ACE format at $300 \mathrm{~K}$. A combination of Bash and Python scripts were used to run MCNP 300 times, each time with a unique set of random files for each isotope, i.e., with a unique representation of the nuclear data. The output was processed and analysed with a set of Python and Matlab scripts and the spread in the MCNP results with respect to the variation in nuclear data was inferred. The uncertainty of the uncertainty was analysed according to the method outlined in [4] under the assumption of Gaussian distributions.

The mean value of the flux and the reaction rates of the 300 random files were also analysed and compared to the results obtained with the FEND2.1 library. i.e., the deviation between TENDL2015 and FENDL2.1 was investigated.

\section{Results}

\section{1. ${ }^{115} \ln \left(n, n^{3}\right)^{115 m} \ln$ effective cross-section uncertainty}

Using the Eq. (2), a value of $\langle\sigma\rangle$ for each random file was calculated, and hence the distribution of $\langle\sigma\rangle$ was derived. $\langle\sigma\rangle$ was calculated with three different spectra: A ${ }^{252} \mathrm{Cf}$ spontaneous fission spectrum, a JET DD and DT spectra at the horizontal entrance (same location as used in [2]). Note that this is not the exact same spectrum that is derived when the transport data uncertainty is calculated. However, the spectra are similar, and this spectrum was chosen to make comparisons to [2] possible. The DD and DT spectra have been computed using MCNP. Figure 3 illustrates the different spectra.

The results for the different spectra are provided in Table 1. The results are compared to what is presented in [2] and in [7]. The one sigma spread is interpreted as the uncertainty and is also reported in Table 1.

\section{Transport data uncertainty}

In this section, the uncertainty due to transport nuclear data for the different reaction rates $(R)$, neutron $(n)$ fluxes and photon $(p)$ fluxes is presented. The uncertainty of the nuclear data uncertainty is also included, referred to as $\Delta$ (STD ND). Furthermore, the difference between the
Table 1. The effective cross-section (mbarn) and its uncertainty derived in this work, in [2] and in [7].

\begin{tabular}{|l|c|c|c|c|}
\hline & \multirow{2}{*}{ This work } & \multirow{2}{*}[2]{} & \multicolumn{2}{|c|}{$[7]$} \\
\cline { 4 - 5 } & & & Experiment & Calculated \\
\hline${ }^{252}$ Cf & 190.5 & 190.6 & 197.4 & 190.6 \\
Spectrum & $(6.6 \pm 0.7 \%)$ & $(1.7 \%)$ & $(1.4 \%)$ & $(1.7 \%)$ \\
\hline JET DD & 76.0 & 76.1 & \multicolumn{2}{|c|}{-} \\
Spectrum & $(7.4 \pm 0.7 \%)$ & $(2.2 \%)$ & \multicolumn{2}{|c|}{} \\
\hline JET DT & 37.4 & 37.5 & \multicolumn{2}{|c|}{-} \\
Spectrum & $(7.2 \pm 0.7 \%)$ & $(1.4 \%)$ & \multicolumn{2}{|c|}{} \\
\hline
\end{tabular}

Table 2. Results for DD and DT plasma using TENDL2015; In column one is the description of the plasma, the location of the MCNP tally with respect to the activation foil, and the observable: $R$ is the reaction rate, $\mathrm{n}$ is the total neutron flux, and $p$ is the total photon flux. Column two presents the difference between TENDL2015 and FENDL2.1 in percent. Column three displays the uncertainty due to TENDL2015 transport nuclear data. Column four shows the uncertainty of the nuclear data uncertainty.

\begin{tabular}{|l|c|c|c|}
\hline & $\begin{array}{c}\text { FENDL vs. } \\
\text { TENDL } \\
(\%)\end{array}$ & $\begin{array}{c}\text { STD ND } \\
(\%)\end{array}$ & $\begin{array}{c}\Delta \text { (STD ND) } \\
(\%)\end{array}$ \\
\hline DD - below - $n$ & 1.3 & 1 & 0.05 \\
\hline DD - above $-n$ & 1.8 & 1.1 & 0.1 \\
\hline DD - below - $R\left({ }^{115} \mathrm{In}\right)$ & 1.8 & 0.8 & 0.04 \\
\hline DD - above - $R\left({ }^{115} \mathrm{In}\right)$ & 3.2 & 1.7 & 0.1 \\
\hline DT - below - $n$ & 2.5 & 2.9 & 0.1 \\
\hline DT - above - $n$ & 2.1 & 3.3 & 0.1 \\
\hline DT - below - $R\left({ }^{115} \mathrm{In}\right)$ & 4.4 & 3.8 & 0.2 \\
\hline DT - above $-R\left({ }^{115} \mathrm{In}\right)$ & 3.7 & 4.5 & 0.2 \\
\hline DT - below - $R\left({ }^{93} \mathrm{Nb}\right)$ & 0.9 & 1.1 & 0.05 \\
\hline DT - above - $R\left({ }^{93} \mathrm{Nb}\right)$ & 2.1 & 2.2 & 0.1 \\
\hline DT - below - p & - & 3.8 & 0.2 \\
\hline DT - above - p & - & 3.4 & 0.2 \\
\hline \multicolumn{2}{|l|}{ Results for fluxes and reaction rates at more locations can be found in $[8]}$.
\end{tabular}

average (of the $\sim 300$ calculations) TENDL2015 results and the FENDL2.1 results are presented.

Since the activation foils are small, the statistical uncertainty for the foils is relative high. Consequently, in Table 2, we instead present the results for some structures close to the activation foils; one structure just below the foils, and the intermediate port which is mostly above the foils.

In Table 2, the results for DT and DD-plasma using TENDL2015 is presented. The reaction rate is investigated for the ${ }^{115} \operatorname{In}\left(n, n^{\prime}\right)^{115 m} \operatorname{In}$ and the ${ }^{93} \mathrm{Nb}(n, 2 n)^{92 m} \mathrm{Nb}$ reaction. Note that in all cases in this table only the uncertainty due to the transport data is analysed and the uncertainty in the activation data is reported in the previous section.

\section{Discussion and conclusion}

For the absolute value, there is a good agreement for the calculated effective cross-section between this work, [2] and [7].

Higher uncertainties are obtained in this work than what is reported in $[2,7]$. The reason for this should be investigated further, but one possible explanation is that there are stronger correlations in the model based TMC 
method than what is available in the IRDFF covariance files used in [2] and [7]. Better ways to include both differential [9] and integral [10] experimental data are currently being developed. Since [2] uses the standard cross-section and its experimental uncertainty directly we would suggest, to use these as the best estimate of the nuclear data uncertainty, i.e., $2.2 \%$ in DD and $1.4 \%$ in DT. However, it can be noted that there is a $3.5 \%$ discrepancy between the experimental results in [7] and what is obtained in the calculations, which is more than what would be expected from the quoted uncertainties in $[2,7]$, so the reaction should be further investigated.

For DD plasmas it is found that the uncertainty in the total neutron flux around the JET activation foil is $\sim 1 \%$ and that the uncertainty in the ${ }^{115} \operatorname{In}\left(n, n^{\prime}\right)^{115 m} \operatorname{In}$ reaction rate is $1-2 \%$. For DT plasmas it is found that the uncertainty in the total neutron flux is $\sim 3 \%$ and that the uncertainty in the ${ }^{115} \operatorname{In}\left(n, n^{\prime}\right)^{115 m}$ In reaction rate is $\sim 4 \%$ and for the ${ }^{93} \mathrm{Nb}(\mathrm{n}, 2 \mathrm{n}){ }^{92 m} \mathrm{Nb}$ reaction rate is $1.1-2,6 \%$. The difference between TENDL2015 average values and FENDL2.1 is typical in the same range as the nuclear data uncertainty.

From this work, it can be concluded the uncertainty due to transport nuclear data needs to be included in the neutron yield uncertainty analysis, in particular for DT plasmas. It is also noted that by using activation reactions with high thresholds, such as the ${ }^{93} \mathrm{Nb}(\mathrm{n}, 2 \mathrm{n})^{92 m} \mathrm{Nb}$ reaction the transport nuclear data uncertainty in DT operation can be significantly reduced. It should be noted that even if TENDL2015 today is the nuclear data library with the most comprehensive covariance information, there are still covariance information that needs to be added, e.g., correlations between different isotopes. There is a continuous effort to improve the TENDL covariance information, and this might either increase or decrease the final uncertainty estimates in the neutron yield estimates.
This work has been carried out within the framework of the EUROfusion Consortium and has received funding from the Euratom research and training programme 2014-2018 under grant agreement No. 633053. The views and opinions expressed herein do not necessarily reflect those of the European Commission.

\section{References}

[1] P. Batistoni, D. Campling, S. Conroy, D. Croft, T. Giegerich, T. Huddleston, X. Lefebvre, I. Lengar, S. Lilley, A. Peacock, M. Pillon, S. Popovichev, S. Reynolds, R. Vila, R. Villari, N. Bekris, Fusion Engineering and Design 109-111, 278-285 (2016)

[2] G. Stankunas, P. Batistoni, S. Conroy, H.Sjöstrand, Nuclear Instruments and Methods in Physics Research Section A 788, 168-172 (2015)

[3] A.J. Koning, D. Rochman, Nuclear Data Sheets 113, 2841-2934 (2012)

[4] D. Rochman, W. Zwermann, SC van der Marck, A. Koning, H. Sjöstrand, P. Helgesson, B. KrzykaczHausmann, Nuclear Science and Engineering 177(3), 337 (2014)

[5] TENDL 2015 (http://www.talys.eu/tendl2015/)

[6] A. Koning, Eur. Phys. J. A 51, 184 (2015)

[7] E.M. Zsolnay, R. Capote, H.J. Nolthenius, A. Trkov, Summary Description of the New International Reactor Dosimetric and Fusion File (IRDFF release 1.0), INDC(NDS)-0616

[8] H. Sjöstrand, EUROfusion Report, WP JET3, Deliverable L11 (2016)

[9] P. Helgesson, H. Sjöstrand, A. Koning, D. Rochman, E. Alhassan, S. Pomp, Nuclear Data Sheets 123, 214219 (2015)

[10] E. Alhassan, H. Sjöstrand, P. Helgesson, M. Österlund, S. Pomp, A.J. Koning, D. Rochman, Progress in Nuclear Energy 88, 43-52 (2016) 